\title{
Properties of Conjugate Fuzzy Matrices based on Reference Function
}

\author{
Mamoni Dhar \\ Assistant Professor, \\ Science College, \\ Kokrajhar, Assam, India
}

\begin{abstract}
In this article, the conjugate of fuzzy matrices based on reference function and some of its properties are taken into consideration. In dealing with these, use the representation of fuzzy matrices with the help of reference function is considered. Thereafter, addition and multiplication are defined accordingly.
\end{abstract}

\section{Keywords}

Reference function, fuzzy matrix, conjugate fuzzy matrix, determinant of fuzzy matrix, transpose of fuzzy matrix.

\section{INTRODUCTION}

Fuzzy matrix has been proposed to represent fuzzy relation in a system based on fuzzy set theory, Ovehinnikov [3]. Fuzzy matrices were introduced first time by Thomason [1], who discussed the convergence of powers of fuzzy matrices. Several authors have presented a number of results on the convergence of power sequence of fuzzy matrices ([2],[4], [5], [6]). It plays an important role in fuzzy set theory. Uses of fuzzy matrices are most common in science and technology. It is well known that the matrix formulation of a mathematical formula gives extra advantage to handle/study the problem under consideration. When some problems are not solved by classical matrices, then the concepts of fuzzy matrices are used. It is important to note here that the representation of fuzzy matrices on the basis of reference function and its various properties have been discussed already in works, $\operatorname{Dhar}([8],[9],[10],[11],[12])$. Here in this article, we shall consider some aspects of conjugate of fuzzy matrices for which it is necessary to define maximum operation, minimum operation, max-min operation and min-max operation on fuzzy matrices when expressed in terms of reference functions as defined by Baruah [7]. We shall deal with these in the following sections.

\section{ADDITION OF FUZZY MATRICES}

Addition of two fuzzy matrices will be discussed in this section. Let us consider two fuzzy matrices A and B as

$$
A=\left[\begin{array}{cccc}
0.3 & 0.7 & 0.8 & 0.9 \\
0.4 & 0.5 & 1 & 0.3 \\
0.6 & 0.1 & 0.4 & 0.8 \\
0.9 & 0.4 & 0.6 & 0.2
\end{array}\right]
$$

and

$$
B=\left[\begin{array}{cccc}
1 & 0.2 & 0.4 & 0.9 \\
0.3 & 0.6 & 0.1 & 0.4 \\
0.8 & 0.9 & 0.5 & 0.6 \\
(0.3 & 0.2 & 0.5 & 0.7
\end{array}\right]
$$

Addition of two fuzzy matrices A and B are compatible under matrix addition if they are of same order. For instance for the fuzzy matrices A and B mentioned above, we have

$$
A+B=\left[\begin{array}{cccc}
1,3 & 0.9 & 1.2 & 1.8 \\
0.7 & 1.1 & 1.1 & 0.7 \\
1.4 & 1 & 0.9 & 1.4 \\
1.2 & 0.6 & 1.1 & 0.9
\end{array}\right]
$$

Here it can be seen that the result is a matrix but not a fuzzy matrix. Hence we see that addition of two fuzzy matrices compatible under addition need not be a fuzzy matrix. It is for this reason the addition should be defined with the help of maximum or minimum operation so that the resulting matrix is a fuzzy matrix.

\subsection{Maximum Operation of Two Fuzzy Matrices}

Two fuzzy matrices are conformable for addition if the matrices are of same order. That is to say, when we wish to find addition of two matrices, the number of rows and columns of both the matrices should be same. If $A=\left[\left(a_{i j}, r_{i j}\right)\right]_{m \times n}$ and $B=\left[\left(b_{i j}, r_{i j}^{\prime}\right)\right]_{m \times n}$ be two matrices of same order then their addition can be defined as follows

$$
A+B=\left\{\max \left(a_{i j}, b_{i j}\right), \min \left(r_{i j}, r_{i j}^{\prime}\right)\right\}
$$

where $a_{i j}$ stands for the membership function of the fuzzy matrix A for the ith row and jth column and $r_{i j}$ is the corresponding reference function and $b_{i j}$ stands for the membership function of the fuzzy matrix B for the $i$ th row and $j$ th column where $r_{i j}^{\prime}$ represents the corresponding reference function.

The two matrices A and B mentioned above when expressed in terms of reference function becomes

$$
A=\left[\begin{array}{cccc}
(0.3,0) & (0.7,0)) & (0.8,0) & (0.9,0) \\
(0.4,0) & (0.5,0) & (1,0) & (0.3,0) \\
(0.6,0) & (0.1,0) & (0.4,0) & (0.8,0) \\
(0.9,0) & (0.4,0) & (0.6,0) & (0.2,0)
\end{array}\right]
$$


$B=\left[\begin{array}{cccc}(1,0) & (0.2,0)) & (0.4,0) & (0.9,0) \\ (0.3,0) & (0.6,0) & (0.1,0) & (0.4,0) \\ (0.8,0) & (0.9,0) & (0.5,0) & (0.6,0) \\ (0.3,0) & (0.2,0) & (0.5,0) & (0.7,0)\end{array}\right]$

Hence for the matrices A and B given above we have

$$
A+B=\max (A, B)
$$

$$
=\left[\begin{array}{cccc}
(1,0) & (0.7,0) & (0.8,0) & (0.9,0) \\
(0.4,0) & (0.6,0) & (1,0) & (0.4,0) \\
(0.8,0) & (0.9,0) & (0.5,0) & (0.8,0) \\
(0.9,0) & (0.4,0) & (0.6,0) & (0.7,0)
\end{array}\right]
$$

\subsection{Minimum Operation of Two Fuzzy Matrices}

If $A=\left[\left(a_{i j}, r_{i j}\right)\right]_{m \times n}$ and $B=\left[\left(b_{i j}, r_{i j}^{\prime}\right)\right]_{m \times n}$ be two matrices of same order then their addition can also be defined as follows

$A+B=\left\{\min \left(a_{i j}, b_{i j}\right), \max \left(r_{i j}, r_{i j}^{\prime}\right)\right\}$

where $a_{i j}$ stands for the membership function of the fuzzy matrix A for the ith row and $j$ th column and $r_{i j}$ is the corresponding reference function and $b_{i j}$ stands for the membership function of the fuzzy matrix B for the $i$ th row and $j$ th column where $r_{i j}^{\prime}$ represents the corresponding reference function.

$$
\min (A, B)=\left[\begin{array}{cccc}
(0.3,0) & (0.2,0) & (0.4,0) & (0.9,0) \\
(0.3,0) & (0.5,0) & (1,0) & (0.3,0) \\
(0.6,0) & (0.1,0) & (0.4,0) & (0.6,0) \\
(0.3,0) & (0.2,0) & (0.5,0) & (0.2,0)
\end{array}\right]
$$

In case of fuzzy matrices, it can be easily seen that the addition is not defined whereas the existence of minimum or maximum operations. Under the minimum or maximum operation the resulting matrix is a fuzzy matrix of same order and hence these operations can be considered for finding the addition of any two fuzzy matrices,

\subsection{Minimum Operation of Two Fuzzy Matrices}

If $A=\left[\left(a_{i j}, r_{i j}\right)\right]_{m \times n}$ and $B=\left[\left(b_{i j}, r_{i j}^{\prime}\right)\right]_{m \times n}$ be two matrices of same order then their addition can also be defined as follows

$$
A+B=\left\{\min \left(a_{i j}, b_{i j}\right), \max \left(r_{i j}, r_{i j}^{\prime}\right)\right\}
$$

where $a_{i j}$ stands for the membership function of the fuzzy matrix A for the ith row and jth column and $r_{i j}$ is the corresponding reference function and $b_{i j}$ stands for the membership function of the fuzzy matrix B for the $i$ th row and $j$ th column where $r_{i j}^{\prime}$ represents the corresponding reference function. Then $A+B$ if calculated by minimum rule defined above will produce the following result.

$$
\begin{aligned}
& A+B=\min (A, B) \\
& =\left[\begin{array}{cccc}
(0.3,0) & (0.2,0) & (0.4,0) & (0.9,0) \\
(0.3,0) & (0.5,0) & (1,0) & (0.3,0) \\
(0.6,0) & (0.1,0) & (0.4,0) & (0.6,0) \\
(0.3,0) & (0.2,0) & (0.5,0) & (0.2,0)
\end{array}\right]
\end{aligned}
$$

In case of fuzzy matrices, it can be easily seen that the addition is not defined whereas the existence of minimum or maximum operations can be found. Under the minimum or maximum operation the resulting matrix is a fuzzy matrix of same order and hence these operations can be considered for finding the addition of any two fuzzy matrices.

\subsection{Product of Two Fuzzy Matrices}

To find the product of two fuzzy matrices A and B mentioned above where these two matrices are conformable for multiplication i.e the number of column of the matrix $\mathrm{A}$ is equal to the number of rows of the second matrix $\mathrm{B}$, the resulting product will not be a fuzzy matrix which is given below

$$
A B=\left[\begin{array}{cccc}
(1.42,0) & (1.38,0) & (1.04,0) & (1.60,0) \\
(1.24,0) & (0.60,0) & (0.86,0) & (1.37,0) \\
(0.74,0) & (0.7,0) & (0.85,0) & (1.38,0) \\
(0.75,0) & (1,0) & (0.8,0) & (1.74,0)
\end{array}\right]
$$

The above result shows that $\mathrm{AB}$ is not a fuzzy matrix. So there is the need of defining a compatible operation for multiplication so that the resulting matrix again becomes a fuzzy matrix. However, even for this new operation if the product $\mathrm{AB}$ is to be defined we need the number of columns of $A$ to be equal to the number of rows of B. Two types of operation which we can have for calculating the product of two fuzzy matrices are max-min operation and min-max operation.

The product of two fuzzy matrices under usual matrix multiplication is not a fuzzy matrix. It is due to this reason; a conformable operation analogous to the product which again happens to be a fuzzy matrix was introduced by many researchers which can be found in fuzzy literature. However, even for this operation if the product $\mathrm{AB}$ to be defined if the number of columns of the first fuzzy matrix is must be equal to the number of rows of the second fuzzy matrix. In the process of finding multiplication of fuzzy matrices, if this condition is satisfied then the multiplication of two fuzzy matrices $\mathrm{A}$ and $\mathrm{B}$, will be defined in the following manner:

$$
A B=\left\{\max \min \left(a_{i j}, b_{j i}\right), \min \max \left(r_{i j}, r_{j i}^{\prime}\right\}\right.
$$

where $A=\left[\left(a_{i j}, r_{i j}\right)\right] \quad$ and $\quad B=\left[\left(b_{i j}, r^{\prime}{ }_{i j}\right)\right]$ $, 1 \leq i \leq n, 1 \leq j \leq n$ and $a_{i j}$, stands for the membership function of the fuzzy matrix $\mathrm{A}$ and the corresponding reference function is $r_{i j}$ whereas $b_{i j}$ stands for the membership function of the fuzzy matrix B with the corresponding reference function $r^{\prime}{ }_{j i}$. This operation of 
fuzzy matrix multiplication is defined in some previous works.

\section{CONJUGATE OF FUZZY MATRICES}

Let $A=\left[\left(a_{i j}, 0\right)\right]$ be a fuzzy matrix , the matrix obtained by replacing each element $\left(a_{i j}, 0\right)$ by $\left(1, a_{i j}\right)$ is called conjugate or complement of the fuzzy matrix of $\mathrm{A}$ and is denoted as $\bar{A} \operatorname{or} A^{c}=\left[\left(1, a_{i j}\right)\right]$.

For example, if

$$
A=\left(\begin{array}{lll}
\left(a_{11}, 0\right) & \left(a_{12}, 0\right) & \left(a_{13}, 0\right) \\
\left(a_{21}, 0\right) & \left(a_{22}, 0\right) & \left(a_{23}, 0\right) \\
\left(a_{31}, 0\right) & \left(a_{32}, 0\right) & \left(a_{33}, 0\right)
\end{array}\right)
$$

be a fuzzy matrix then its conjugate will be expressed in the

form

$$
C=\left(\begin{array}{lll}
\left(1, a_{11}\right) & \left(1, a_{12}\right) & \left(1, a_{13}\right) \\
\left(1, a_{21}\right) & \left(1, a_{22}\right) & \left(1, a_{23}\right) \\
\left(1, a_{31}\right) & \left(1, a_{32}\right) & \left(1, a_{33}\right)
\end{array}\right)
$$

The multiplication of two fuzzy matrices which are conformable for multiplication are discussed below

\subsection{Multiplication of Conjugate of Fuzzy Matrices}

Let $\mathrm{C}$ and $\mathrm{D}$ be two fuzzy matrices such that

$C=\left(\begin{array}{lll}\left(1, a_{11}\right) & \left(1, a_{12}\right) & \left(1, a_{13}\right) \\ \left(1, a_{21}\right) & \left(1, a_{22}\right) & \left(1, a_{23}\right) \\ \left(1, a_{31}\right) & \left(1, a_{32}\right) & \left(1, a_{33}\right)\end{array}\right)$

and

$$
D=\left(\begin{array}{lll}
\left(1, b_{11}\right) & \left(1, b_{12}\right) & \left(1, b_{13}\right) \\
\left(1, b_{21}\right) & \left(1, b_{22}\right) & \left(1, b_{23}\right) \\
\left(1, b_{31}\right) & \left(1, b_{32}\right) & \left(1, b_{33}\right)
\end{array}\right)
$$

be two fuzzy matrices. Then the multiplication of these two matrices would be defined in our way as

$$
C D=\left(\begin{array}{lll}
E_{11} & E_{12} & E_{13} \\
E_{21} & E_{22} & E_{23} \\
E_{31} & E_{32} & E_{33}
\end{array}\right)
$$

where

$$
\begin{aligned}
& E_{11}=[\max \{\min (1,1), \min (1,1), \min (1,1)\}, \\
& \left.\min \left\{\max \left(a_{11}, b_{11}\right), \max \left(a_{12}, b_{21}\right), \max \left(a_{13}, b_{31}\right)\right\}\right]
\end{aligned}
$$

$E_{12}=[\max \{\min (1,1), \min (1,1), \min (1,1)\}$, $\left.\min \left\{\max \left(a_{11}, b_{12}\right), \max \left(a_{12}, b_{22}\right), \max \left(a_{13}, b_{23}\right)\right\}\right]$

$E_{21}=[\max \{\min (1,1), \min (1,1), \min (1,1)\}$, $\left.\min \left\{\max \left(a_{21}, b_{11}\right), \max \left(a_{22}, b_{21}\right), \max \left(a_{23}, b_{31}\right)\right\}\right]$ $E_{22}=[\max \{\min (1,1), \min (1,1), \min (1,1)\}$, $\min \left\{\max \left(a_{21}, b_{12}\right), \max \left(a_{22}, b_{22}\right), \max \left(a_{23}, b_{32}\right\}\right]$ $E_{23}=[\max \{\min (1,1), \min (1,1), \min (1,1)\}$, $\min \left\{\max \left(a_{21}, b_{13}\right), \max \left(a_{22}, b_{23}\right), \max \left(a_{23}, b_{33}\right\}\right]$

Then the multiplication of these two matrices if min-max operation would be defined in our way as

$$
C D=\left(\begin{array}{lll}
D_{11} & D_{12} & D_{13} \\
D_{21} & D_{22} & D_{23} \\
D_{31} & D_{32} & D_{33}
\end{array}\right)
$$

where

$D_{11}=[\min \{\max (1,1), \max (1,1), \max (1,1)\}$, $\left.\max \left\{\min \left(a_{11}, b_{11}\right), \min \left(a_{12}, b_{21}\right), \min \left(a_{13}, b_{31}\right)\right\}\right]$

$D_{12}=[\min \{\max (1,1), \max (1,1), \max (1,1)\}$, $\left.\max \left\{\min \left(a_{11}, b_{12}\right), \min \left(a_{12}, b_{22}\right), \min \left(a_{13}, b_{23}\right)\right\}\right]$

$D_{21}=[\min \{\max (1,1), \max (1,1), \max (1,1)\}$, $\left.\max \left\{\min \left(a_{21}, b_{11}\right), \min \left(a_{22}, b_{21}\right), \min \left(a_{23}, b_{31}\right)\right\}\right]$

$D_{22}=[\min \{\max (1,1), \max (1,1), \max (1,1)\}$, $\max \left\{\min \left(a_{21}, b_{12}\right), \min \left(a_{22}, b_{22}\right), \min \left(a_{23}, b_{32}\right\}\right]$

$D_{23}=[\min \{\max (1,1), \max (1,1), \max (1,1)\}$, $\max \left\{\min \left(a_{21}, b_{13}\right), \min \left(a_{22}, b_{23}\right), \min \left(a_{23}, b_{33}\right\}\right]$

\subsection{Properties of Multiplication of Conjugate Fuzzy Matrices}

Conjugate fuzzy matrices A and B satisfies De Morgan's rule under fuzzy matrix product of max-min and min-max operation as given below:

(i) $\overline{\max -\min (A B)}=\min -\max (\bar{A} \bar{B})$

(ii) $\overline{\min -\max (A B)}=\max -\min (\bar{A} \bar{B})$

If two fuzzy matrices $\mathrm{A}$ and $\mathrm{B}$ mentioned above are considered for proving the rules, the product $\mathrm{AB}$ can be calculated as follows: 
$\max -\min (A B)$

$=\left[\begin{array}{llll}(0.8,0) & (0.8,0) & (0.5,0) & (0.7,0) \\ (0.8,0) & (0.9,0) & (0.5,0) & (0.6,0) \\ (0.6,0) & (0.4,0) & (0.5,0) & (0.7,0) \\ (0.9,0) & (0.6,0) & (0.5,0) & (0.9,0)\end{array}\right]$

Then we have the conjugate of this matrix as

$$
\begin{aligned}
& \overline{\max -\min (A B)} \\
& =\left[\begin{array}{llll}
(1,0.8) & (1,0.8) & (1,0.5) & (1,0.7) \\
(1,0.8) & (1,0.9) & (1,0.5) & (1,0.6) \\
(1,0.6) & (1,0.4) & (1,0.5) & (1,0.7) \\
(1,0.9) & (1,0.6) & (1,0.5) & (1,0.9)
\end{array}\right]
\end{aligned}
$$

Now we shall calculate the product of the conjugate of fuzzy matrices A and B with the help of min-max operation

$$
\begin{aligned}
\widehat{A} & =\left[\begin{array}{cccc}
(1,0.3) & (1,0.7) & (1,0.8) & (1,0.9) \\
(1,0.4) & (1,0.5) & (1,1) & (1,0.5) \\
(1,0.6) & (1,0.1) & (1,0.4) & (1,0.8) \\
(1,0.9) & (1,0.4) & (1,0.6) & (1,0.2)
\end{array}\right] \\
\bar{B} & =\left[\begin{array}{cccc}
(1,1) & (1,0.2) & (1,0.4) & (1,0.9) \\
(1,0.3) & (1,0.6) & (1,0.1) & (1,0.4) \\
(1,0.8) & (1,0.9) & (1,0.5) & (1,0.6) \\
(1,0.3) & (1,0.2) & (1,0.5) & (1,0.7)
\end{array}\right]
\end{aligned}
$$

Then the product $\bar{A} \bar{B}$ can be calculated as $\bar{A} \bar{B}=\left(d_{i j}\right)$ where

$d_{11}=[\min \{\max (1,1), \max (1,1), \max (1,1), \max (1,1)\}$, $\max \{\min (0.3,1), \min (0.7,0.3)$,

$\min (0.8,0.8), \min (0.9,0.3)]$

$=[\min (1,1,1,1), \max (0.3,0.3,0.8,0.3)]$

$=(1,0.8)$

$d_{12}=[\min \{\max (1,1), \max (1,1), \max (1,1), \max (1,1)\}$, $\max \{\min (0.3,0.2), \min (0.7,0.6)$,

$\min (0.8,0.9), \min (0.9,0.2)]$

$=[\min (1,1,1,1), \max (0.2,0.6,0.8,0.2)]$

$=(1,0.8)$

$d_{13}=[\min \{\max (1,1), \max (1,1), \max (1,1), \max (1,1)\}$,

$\max \{\min (0.3,0.4), \min (0.7,0.1)$,

$\min (0.8,0.5), \min (0.9,0.5)]$

$=[\min (1,1,1,1), \max (0.3,0.1,0.5,0.5)]$
$=(1,0.5)$

$d_{14}=[\min \{\max (1,1), \max (1,1), \max (1,1), \max (1,1)\}$, $\max \{\min (0.3,0.9), \min (0.7,0.4)$, $\min (0.8,0.6), \min (0.9,0.7)]$

$=[\min (1,1,1,1), \max (0.3,0.4,0.6,0.7)]$

$=(1,0.7)$

$d_{21}=[\min \{\max (1,1), \max (1,1), \max (1,1), \max (1,1)\}$, $\max \{\min (0.4,1), \min (0.5,0.3)$,

$\min (0.1,0.8), \min (0.3,0.3)]$

$=\{\min (1,1,1,1), \max (0.4,0.3,0.1,0.3)]$

$=(1,0.4)$

$d_{22}=[\min \{\max (1,1), \max (1,1), \max (1,1), \max (1,1)\}$, $\max \{\min (0.4,0.2), \min (0.5,0.6)$,

$\min (0.9,1), \min (0.3,0.2)]$

$=[\min (1,1,1,1), \max (0.2,0.5,0.9,0.2)]$

$=(1,0.9)$

$d_{23}=[\min \{\max (1,1), \max (1,1), \max (1,1), \max (1,1)\}$, $\max \{\min (0.4,0.4), \min (0.5,0.1)$,

$\min (0.9,0.5), \min (0.3,0.5)]$

$=[\min (1,1,1,1), \max (0.4,0.1,0.5,0.3)]$

$=(1,0.5)$

$d_{24}=[\min \{\max (1,1), \max (1,1), \max (1,1), \max (1,1)\}$, $\max \{\min (0.4,0.9), \min (0.5,0.4)$,

$\min (0.9,0.6), \min (0.3,0.7)]$

$=[\min (1,1,1,1), \max (0.4,0.4,0.6,0.3)$

$=(1,0.6)]$

$d_{31}=[\min \{\max (1,1), \max (1,1), \max (1,1), \max (1,1)\}$, $\max \{\min (0.6,1), \min (0.1,0.3)$,

$\min (0.4,0.8), \min (0.8,0.3)]$

$=[\min (1,1,1,1), \max (0.6,0.1,0.4,0.3)]$

$=(1,0.6)$

$d_{32}=[\min \{\max (1,1), \max (1,1), \max (1,1), \max (1,1)\}$, $\max \{\min (0.6,0.2), \min (0.1,0.6)$,

$\min (0.4,1), \min (0.8,0.2)]$

$=[\min (1,1,1,1), \max (0.2,0.1,0.4,0.2)]$

$=(1,0.4)$

$d_{32}=[\min \{\max (1,1), \max (1,1), \max (1,1), \max (1,1)\}$, $\max \{\min (0.6,0.4), \min (0.1,0.1)$,

$\min (0.4,0.5), \min (0.8,0.5)]$

$=[\min (1,1,1,1), \max (0.4,0.1,0.4,0.5)]$

$=(1,0.5)$ 
$d_{33}=[\min \{\max (1,1), \max (1,1), \max (1,1), \max (1,1)\}$, $\max \{\min (0.6,0.9), \min (0.1,0.4)$,

$\min (0.4,0.6), \min (0.8,0.7)]$

$=[\min (1,1,1,1), \max (0.6,0.1,0.4,0.7)]$

$=(1,0.7)$

$d_{34}=[\min \{\max (1,1), \max (1,1), \max (1,1), \max (1,1)\}$, $\max \{\min (0.6,0.4), \min (0.1,0.1)$,

$\min (0.4,0.5), \min (0.8,0.5)]$

$=[\min (1,1,1,1), \max (0.4,0.1,0.4,0.5)]$

$=(1,0.5)$

and so on.

Thus we see that

$\overline{\max -\min (A B)}=\min -\max (\bar{A} \bar{B})$

Now if A and B be two fuzzy matrices then

$\min -\max (\mathrm{AB})$

$=\left[\begin{array}{llll}(0.7,0) & (0.3,0) & (0.4,0) & (0.7,0) \\ (0.3,0) & (0.3,0) & (0.4,0) & (0.5,0) \\ (0.3,0) & (0.6,0) & (0.1,0) & (0.4,0) \\ (0.3,0) & (0.2,0) & (0.4,0) & (0.4,0)\end{array}\right]$

$\overline{\min -\max (A B)}$

$=\left[\begin{array}{llll}(1,0.7) & (1,0.3) & (1,0.4) & (1,0.7) \\ (1,0.3) & (1,0.3) & (1,0.4) & (1,0.5) \\ (1,0.3) & (1,0.6) & (1,0.1) & (1,0.4) \\ (1,0.3) & (1,0.2) & (1,0.4) & (1,0.4)\end{array}\right]$

Then the product $\bar{A} \bar{B}$ can be calculated as $\bar{A} \bar{B}=\left(d_{i j}\right)$ where

$d_{11}=[\max \{\min (1,1), \min (1,1), \min (1,1), \min (1,1)\}$, $\min \{\max (0.3,1), \max (0.7,0.3)$,

$\max (0.8,0.8), \max (0.9,0.3)]$

$=\{\max (1,1,1,1), \min (1,0.7,0.8,0.9)\}$

$=(1,0.7)$

$d_{12}=[\max \{\min (1,1), \min (1,1), \min (1,1), \min (1,1)\}$,

$\min \{\max (0.3,0.2), \max (0.7,0.6)$,

$\max (0.8,0.9), \max (0.9,0.2)]$

$d_{13}=[\max \{\min (1,1), \min (1,1), \min (1,1), \min (1,1)\}$,

$\min \{\max (0.3,0.4), \max (0.7,0.1)$,

$\max (0.8,0.5), \max (0.9,0.5)]$ $d_{14}=[\max \{\min (1,1), \min (1,1), \min (1,1), \min (1,1)\}$,

$\min \{\max (0.3,0.9), \max (0.7,0.4)$,

$\max (0.8,0.6), \max (0.9,0.7)]$

$d_{21}=[\max \{\min (1,1), \min (1,1), \min (1,1), \min (1,1)\}$, $\min \{\max (0.4,1), \max (0.5,0.3)$,

$\max (0.1,0.8), \max (0.3,0.3)]$

$d_{22}=[\max \{\min (1,1), \min (1,1), \min (1,1), \min (1,1)\}$, $\min \{\max (0.4,0.2), \max (0.5,0.6)$,

$\max (0.9,1), \max (0.3,0.2)]$

$d_{23}=[\max \{\min (1,1), \min (1,1), \min (1,1), \min (1,1)\}$, $\max \{\max (0.4,0.4), \max (0.5,0.1)$,

$\max (0.9,0.5), \max (0.3,0.5)]$

$d_{24}=[\max \{\min (1,1), \min (1,1), \min (1,1), \min (1,1)\}$, $\min \{\max (0.4,0.9), \max (0.5,0.4)$,

$\max (0.9,0.6), \max (0.3,0.7)]$

$d_{31}=[\max \{\min (1,1), \min (1,1), \min (1,1), \min 1,1)\}$, $\min \{\max (0.6,1), \max (0.1,0.3)$,

$\max (0.4,0.8), \max (0.8,0.3)]$

$d_{32}=[\max \{\min (1,1), \min (1,1), \min (1,1), \min (1,1)\}$, $\min \{\max (0.6,0.2), \max (0.1,0.6)$,

$\max (0.4,1), \max (0.8,0.2)]$

$d_{32}=[\max \{\min (1,1), \min (1,1), \min (1,1), \min (1,1)\}$, $\min \{\max (0.6,0.4), \max (0.1,0.1)$, $\max (0.4,0.5), \max (0.8,0.5)]$

$d_{33}=[\max \{\min (1,1), \min (1,1), \min (1,1), \min (1,1)\}$, $\min \{\max (0.6,0.9), \max (0.1,0.4)$, $\max (0.4,0.6), \max (0.8,0.7)]$

$d_{32}=[\max \{\min (1,1), \min (1,1), \min (1,1), \min (1,1)\}$, $\min \{\max (0.6,0.2), \max (0.1,0.6)$, $\max (0.4,1), \max (0.8,0.2)]$

and so on.

Continuing the process, it can be seen that

$\max -\min (\bar{A} \bar{B})$

$=\left[\begin{array}{llll}(1,0.7) & (1,0.3) & (1,0.4) & (1,0.7) \\ (1,0.3) & (1,0.3) & (1,0.4) & (1,0.5) \\ (1,0.3) & (1,0.6) & (1,0.1) & (1,0.4) \\ (1,0.3) & (1,0.2) & (1,0.4) & (1,0.4)\end{array}\right]$

Thus it can be concluded that the conjugate of fuzzy matrices when expressed in terms of reference function satisfies De 
Morgan's rule of multiplication of fuzzy matrices when calculated in terms of max-min or min-max operations.

\subsection{Properties of Conjugate Fuzzy \\ Matrices with Determinant and \\ Transpose}

The other properties which can also be found for conjugate fuzzy matrices are the following

$$
\begin{array}{ll}
\text { i. } & \overline{A^{T}}=(\bar{A})^{T} \\
\text { ii. } & \operatorname{det}(\bar{A})^{T}=\operatorname{det} \bar{A}
\end{array}
$$

where $A^{T}$ stands for the transpose of the fuzzy matrix A.

Let a fuzzy matrix be of the following form

$$
A=\left[\begin{array}{ccc}
(0.5,0) & (0.6,0) & (0,0) \\
(0.3,0) & (0.2,0) & (0.7,0) \\
(0.8,0) & (0.9,0) & (0.4,0)
\end{array}\right]
$$

Then the transpose of this fuzzy matrix A is as follows

$$
A^{T}=\left[\begin{array}{ccc}
(0.5,0) & (0.3,0) & (0.8,0) \\
(0.6,0) & (0.2,0) & (0.9,0) \\
(0,0) & (0.7,0) & (0.4,0)
\end{array}\right]
$$

Then the conjugate of this transpose is

$$
\left(\overline{A^{T}}\right)=\left[\begin{array}{ccc}
(1,0.5) & (1,0.3) & (1,0.8) \\
(1,0.6) & (1,0.2) & (1,0.9) \\
(1,0) & (1,0.7) & (1,0.4)
\end{array}\right]
$$

$$
\begin{aligned}
& \text { Again } \\
& \bar{A}=\left[\begin{array}{ccc}
(1,0.5) & (1,0.6) & (1,0) \\
(1,0.3) & (1,0.2) & (1,0.7) \\
(1,0.8) & (1,0.9) & (1,0.4)
\end{array}\right]
\end{aligned}
$$

Then the transpose of this conjugate fuzzy matrix is

$$
(\bar{A})^{T}=\left[\begin{array}{ccc}
(1,0.5) & (1,0.3) & (1,0.8) \\
(1,0.6) & (1,0.2) & (1,0.9) \\
(1,0) & (1,0.7) & (1,0.4)
\end{array}\right]
$$

Hence it can be observed that in case of fuzzy matrices

$$
\overline{A^{T}}=(\bar{A})^{T}
$$

The determinant of the conjugate of the fuzzy matrix $\mathrm{A}$ is as follows

$$
\begin{aligned}
& \operatorname{det} \bar{A}=\left|\begin{array}{ccc}
(1,0.5) & (1,0.6) & (1,0) \\
(1,0.3) & (1,0.2) & (1,0.7) \\
(1,0.8) & (1,0.9) & (1,0.4)
\end{array}\right| \\
& =(1,0.5)\left|\begin{array}{ll}
(1,0.2) & (1,0.7) \\
(1,0.9) & (1,0.4)
\end{array}\right| \\
& +(1,0.6)\left|\begin{array}{ll}
(1,0.3) & (1,0.7) \\
(1,0.8) & (1,0.4)
\end{array}\right| \\
& +(1,0)\left|\begin{array}{ll}
(1,0.3) & (1,0.2) \\
(1,0.8) & (1,0.9)
\end{array}\right| \\
& =(1,0.5)[\max \{\min (1,1), \min (1,1)\} \text {, } \\
& \min \{\max (0.2,0.4), \max (0.7,0.9)\} \\
& +(1,0.6)[\max \{\min (1,1), \min (1,1)\} \text {, } \\
& \min \{\max (0.3,0.4), \max (0.7,0.8)\}+(1,0.8)[\max \{\min (1,1), \\
& \min (1,1)\}, \min \{\max (0.3,0.9), \max (0.2,0.8)\} \\
& =(1,0.5)[\max (1,1), \min (0.4,0.9)]+(1,0.6)[\max (1,1) \text {, } \\
& \min (0.4,0.8)]+(1,0.8)[\max (1,1), \min (0.9,0.8)] \\
& =(1,0.5)(1,0.4)+(1,0.6)(1,0.4)+(1,0.8)(1,0.8) \\
& =(1,0.5)+(1,0.6)+(1,0.8) \\
& =(1,0.5)+(1,0.8) \\
& =(1,0.5)
\end{aligned}
$$

The determinant of the conjugate transpose of this fuzzy matrix is

$$
\begin{aligned}
& \operatorname{det}(\bar{A})^{T}=\left[\begin{array}{ccc}
(1,0.5) & (1,0.3) & (1,0.8) \\
(1,0.6) & (1,0.2) & (1,0.9) \\
(1,0) & (1,0.7) & (1,0.4)
\end{array}\right] \\
& =(1,0.5)\left|\begin{array}{cc}
(1,0.2) & (1,0.9) \\
(1,0.7) & (1,0.4)
\end{array}\right| \\
& +(1,0.3)\left|\begin{array}{cc}
(1,0.6) & (1,0.9) \\
(1,0) & (1,0.4)
\end{array}\right| \\
& +(1,0.8)\left|\begin{array}{cc}
(1,0.6) & (1,0.2) \\
(1,0) & (1,0.7)
\end{array}\right|
\end{aligned}
$$


$=(1,0.5)[\max \{\min (1,1), \min (1,1)\}$,

$\min \{\max (0.2,0.4), \max (0.7,0.9)\}]+(1,0.3)[\max \{\min (1,1)$, $\min (1,1)\}, \min \{\max (0.6,0.4), \max (0,0.9)\}]+(1$,

$0.8)[\max \{\min (1,1), \min (1,1)\}, \min \{\max (0.6,0.7)$,

$\max (0.2,0)\}]$

$=(1,0.5)(1,0.4)+(1,0.3)(1,0.6)+(1,0.8)(1,0.2)$

$=(1,0.5)+(1,0.6)+(1,0.8)$

$=(1,0.5)+(1,0.8)$

$=(1,0.5)$

Hence $\operatorname{det}(\bar{A})^{T}=\operatorname{det} \bar{A}$

\section{CONCLUSION}

Here it can be concluded that fuzzy matrices when represented with the help of reference function and its conjugate defined accordingly along with a suitable definition of addition and multiplication satisfies De Morgan's rule under fuzzy matrix product. Further some other properties of the conjugate fuzzy matrices are also discussed in this article.

\section{REFERENCES}

[1] Thomason, M.G., Convergence of powers of a fuzzy matrix, Journal of Mathematical Analysis and Applications, 57, 476-480, Elsevier, 1977.

[2] Kim, J.B., Determinant theory for Fuzzy and Boolean Matices, Congressus Numerantium Utilitus Mathematica Pub, 273-276, 1978.
[3] Ovehinnikov, S.V., Structure of fuzzy relations, Fuzzy Sets and Systems, 6, 169-195, 1981.

[4] Kim, J.B., Inverses of Boolean Matrices, Bull.Inst. Math. Acod, Science 12(2), 125-128, 1984.

[5] Kim, J.B., Idempotents and Inverses in Fuzzy Matrices, Malayasian Math 6(2), Management Science, 1988.

[6] Kim, J.B., and Baartmans., Determinant Theory for Fuzzy Matrices, Fuzzy Sets and Systems, 29, 349-356, 1989.

[7] Baruah, H. K., Theory of Fuzzy sets Beliefs and Realities, International Journal of Energy, Information and Communications, 2(2), 1-22, 2011.

[8] Dhar, M., Representation of fuzzy matrices Based on Reference Function, International Journal of Intelligent systems and Applications, 5(2), 84-90, 2013.

[9] Dhar, M., A Note on Determinant and Adjoint of Fuzzy Square Matrix , International Journal of Intelligent systems and Applications, 5(5), 58-67, 2013

[10] Dhar, M., A note on Determinant of square fuzzy matrix, International Journal of Information Engineering and Electronic Business, 5(1),26-32, 2013

[11] Dhar, M., A note on fuzzy relational matrices, International Journal of Intelligent systems and Applications, 5(10), 43-49, 2013.

[12] Dhar, M., On Fuzzy Soft matrix based on reference function, International Journal of Information Engineering and Electronic Business, 5(2), 52-59, 2013. 